(2) Open Access Full Text Article

\title{
Development and in vitro characterization of poly(lactide-co-glycolide) microspheres loaded with an antibacterial natural drug for the treatment of long-term bacterial infections
}

\author{
This article was published in the following Dove Press journal: \\ Drug Design, Development and Therapy \\ 7 September 2016 \\ Number of times this article has been viewed
}

\section{Jochen Reinbold' \\ Teresa Hierlemann' \\ Helena Hinkel' \\ Ingrid Müller ${ }^{2}$ \\ Martin E Maier ${ }^{3}$ \\ Tobias Weindl ${ }^{4}$ \\ Christian Schlensak' \\ Hans Peter Wendel' \\ Stefanie Krajewski'}

'Department of Thoracic, Cardiac and Vascular Surgery, University Hospital

Tuebingen, Tuebingen, ${ }^{2}$ Department of Pharmaceutical Engineering, AlbstadtSigmaringen University, Sigmaringen, ${ }^{3}$ Institute of Organic Chemistry, University Tuebingen, Tuebingen, ${ }^{4}$ Aimecs $\mathrm{GmbH}$, Pfarrkirchen, Germany
Correspondence: Stefanie Krajewski Department of Thoracic, Cardiac and Vascular Surgery, University Hospital

Tuebingen, Calwerstr 7/I, 72076

Tuebingen, Germany

Tel +49 707I 2983303

Fax +49 707I 293617

Email stefanie.krajewski@

uni-tuebingen.de

\begin{abstract}
Biodegradable polymers, especially poly(lactide-co-glycolide) (PLGA), have good biocompatibility and toxicological properties. In combination with active ingredients, a specialized drug delivery system can be generated. The aim of the present study was to develop a drug delivery system consisting of PLGA microspheres loaded with the natural active ingredient totarol, which has several antimicrobial mechanisms. Totarol, isolated from the Podocarpus totara tree, was purified using column chromatography, and the eluate was checked for purity using thin layer chromatography. The spherically shaped microspheres with mean diameters of $147.21 \pm 3.45 \mu \mathrm{m}$ and 131.14 $\pm 3.69 \mu \mathrm{m}$ (totarol-loaded and -unloaded microspheres, respectively) were created using the single emulsion evaporation method. Furthermore, the encapsulation efficiency, in a range of $84.72 \% \pm 6.68 \%$ to $92.36 \% \pm 0.99 \%$, was measured via UV/vis spectroscopy. In a 90-day in vitro drug release study, the release of totarol was investigated by $\mathrm{UV} /$ vis spectroscopy as well, showing a release of $53.76 \%$. The toxicity on cells was determined using BJ fibroblasts or Human Embryonic Kidney cells and an 3-(4,5-dimethylthiazol-2-yl)2,5-diphenyltetrazolium bromide assay, which showed no influence on the cell growth. The minimal inhibitory concentration was ascertained. A totarol concentration between $64 \mu \mathrm{g} / \mathrm{mL}$ and $128 \mu \mathrm{g} / \mathrm{mL}$ was necessary to inhibit the bacterial growth over a period of 24 hours. Biofilm formation on the surface of totarol-loaded microspheres was determined using transmission electron microscopy. No biofilm formation could be detected, even if the totarol concentration was below the minimal inhibitory concentration. The hemocompatibility investigations on various markers with fresh heparinized blood $(1.5 \mathrm{IU} / \mathrm{mL})$ showed that totarol and totarol-loaded microspheres have no influence on different blood parameters. The PLGA microspheres characterized by slow release of totarol and great entrapment efficiency represent a novel drug delivery system, which may be highly beneficial for the long-term therapy of bacterial infections.
\end{abstract}

Keywords: totarol, drug delivery system, Staphylococcus aureus, antibacterial, cytotoxicity

\section{Introduction}

In recent decades, biodegradable polymers, especially poly(lactide-co-glycolide) (PLGA), have been used as drug delivery systems in medicine and pharmacy, mainly due to their good biodegradability, biocompatibility, and toxicological properties. Moreover, the amount of encapsulated agents as well as their release and biological distribution can be controlled. ${ }^{1-3}$

PLGA is one of the few polymers that have been approved by the FDA for clinical use in humans. Nowadays, PLGA is very widely used in drug delivery systems with 
controlled drug release over a few days to months, including microspheres, nanoparticles, and implant coatings for local delivery. ${ }^{4,5}$ Besides the release rate, which is affected by particle size and degradation behavior, the inclusion efficiency of the active ingredients plays a pivotal role.

Many different protocols exist for the production of microspheres, for example, different evaporation methods, phase separation methods, and spraying methods. ${ }^{6}$ One widely used method is the solvent evaporation method. ${ }^{7}$ This variant can be divided into four steps. First, the PLGA and the active ingredient are solved in an organic solvent. Second, this organic phase is emulsified with a second, usually aqueous, phase. These two phases are immiscible. Third, the organic solvent is evaporated, and the resulting preemulsion droplets harden. Finally, the resulting microspheres are separated from the liquid and dried. ${ }^{8}$

Next to antibiotics, naturally occurring agents exhibiting antibacterial activity have great potential in prophylactic therapy, since antibiotic resistances may be excluded. The active ingredient totarol, a taxane, is a natural substance extracted from the Totara tree. Kubo et $\mathrm{al}^{9}$ extracted six different diterpenes from the bark of Podocarpus totara and investigated their effect on microorganisms, and only totarol showed antimicrobial effects. Several mechanisms for the effect of totarol were found, including inhibition of energycoupled respiratory transport, prevention of peroxidation of unsaturated fatty acids in the lipid bilayer, and hampering of oxidative phosphorylation by inhibiting the crucial enzymes. However, the exact antimicrobial mechanism is not entirely clear. Further studies have described the antimicrobial effect of totarol on $\beta$-lactam-resistant bacteria strains. ${ }^{10}$ This property is very interesting and may be helpful for future antimicrobial therapies, since totarol has low cytotoxicity. ${ }^{11,12}$

The aim of our study is to embed the water-insoluble active ingredient totarol in the highest possible concentration in PLGA microspheres. Therefore, the single emulsion method and the double emulsion method were applied.

The combination of totarol and slowly degrading microspheres is ideal for the coating of implants, in order to prevent implant-related infections caused by bacterial biofilm formation.

\section{Materials and methods Materials}

PLGA (Resomer ${ }^{\circledR}$ RG 502H MW 7,000-17,000) was purchased from Evonik Industries AG (Essen, Germany), while polyvinyl alcohol (PVA, Mowiol $\left.{ }^{\circledR} 18-88, \mathrm{MW} \sim 130,000\right)$, ethyl acetate (ACS grade), Brij ${ }^{\circledR} 30$ (polyoxyethylene [4] lauryl ether), sodium chloride $(\mathrm{NaCl})$, cerium (IV) sulfate, ammonium molybdate tetrahydrate, hexane, and Mueller Hinton broth were purchased from Sigma-Aldrich Co. (St Louis, MO, USA). Mueller Hinton Blood agar plates were purchased from Oxoid Deutschland GmbH (Wesel, Germany). The active ingredient "totarol" was provided by Aimecs ${ }^{\circledR} \mathrm{GmbH}$ (Pfarrkirchen, Germany). Dimethyl sulfoxide (DMSO) and petroleum ether were purchased from EMD Millipore (Billerica, MA, USA). 3-(4,5-Dimethylthiazol-2yl)-2,5-diphenyltetrazolium bromide (MTT) was purchased from AppliChem (Darmstadt, Germany), and phosphatebuffered saline (PBS), Dulbecco's Modified Eagle's Medium (DMEM), high glucose (with 10\% fetal bovine serum, 4.5 mmol L-glutamine, $30 \mathrm{mmol}$ 4-(2-hydroxyethyl)-1piperazineethanesulfonic acid [HEPES], and 5\% penicillinstreptomycin), and Roswell Park Memorial Institute (RPMI) without phenol red media were purchased from Thermo Fisher Scientific (Waltham, MA, USA). Silica gel (Silica $60 \mathrm{M}, 0.04-0.063 \mathrm{~mm}$ ) and thin layer chromatography (TLC) plates were purchased from Macherey Nagel (Düren, Germany).

\section{Totarol purification}

The active ingredient totarol was obtained with a purity of $\sim 68 \%$. In order to use totarol in medicine or drug products, it was imperative to purify this substance before use.

For purification, $2.5 \mathrm{~g}$ of totarol were dissolved in $5 \mathrm{~mL}$ ethyl acetate, which was loaded on top of a silica gel column. The column was manually packed using a frit and the slurry method. The eluting solvent used was a combination of petroleum ether and ethyl acetate in a ratio of 20:1 with an applied pressure of 0.06 mbar. Fractions were collected in test tubes graduated with the fraction volume of $10 \mathrm{~mL}$. Each of the fractions was visualized by spotting on TLC plates using a heat-sensitive reagent (molybdenum/cerium solution) and air by $350^{\circ} \mathrm{C}$ (using a heat gun). The fractions containing almost pure totarol were added together and placed under a fume hood where the liquid part vaporized. The purified totarol was redissolved in $2 \mathrm{~mL}$ of hexane at $40^{\circ} \mathrm{C}$ and recrystallized by gradually cooling down to $-25^{\circ} \mathrm{C}$. Purification control was done by TLC.

\section{Preparation of totarol-loaded microspheres}

To prepare microspheres at a laboratory scale, the evaporation method was used. Here, the microspheres were formed in a stable oil in water $(\mathrm{o} / \mathrm{w})$ emulsion. To produce this emulsion, two different phases were needed: the continuous phase (CP) and the dispersed phase (DP). The CP contained $\mathrm{NaCl}$ and 1\% PVA as an emulsifier dissolved in distilled water. 
The DP included PLGA, totarol, and polyoxyethylene (4) lauryl ether as an emulsifier dissolved in ethyl acetate.

Preparation of the CP: The CP was created by adding $\mathrm{NaCl}$ and PVA to distilled water under stirring and heating. The solution had to cool down to room temperature before use.

Preparation of the DP: PLGA and totarol were dissolved under stirring in ethyl acetate. Under continuous stirring, polyoxyethylene (4) lauryl ether was added.

To emulsify the two phases, the DP was pipetted carefully into the $\mathrm{CP}$ to create a stable emulsion. Different parameters during the emulsification (eg, stirring and pipetting speed) had to be observed. After several time intervals, the stirring speed was reduced. Under vaporization of ethyl acetate, the curing of microspheres started. The hardening process ended when ethyl acetate evaporated completely.

Phase merging o/w: The DP was injected uniformly at $1,000 \mathrm{rpm}$ into the CP at a ratio of 1:40. After completely injecting the DP, the stirring speed was reduced to $600 \mathrm{rpm}$ for 60 seconds and then to $350 \mathrm{rpm}$ for 4 hours. At this time, the ethyl acetate was completely evaporated.

Subsequently, the created microspheres were washed three times, separated by filtration, and air dried.

Unloaded microspheres were created without the addition of totarol to the DP.

\section{Microscopy}

The microspheres were examined with an optical microscope (Nikon Eclipse E600; Nikon Corporation, Tokyo, Japan) equipped with a PixeLINK camera (PixeLINK, Ottawa, ON, Canada) and with a scanning electron microscope (Zeiss LEO 1430; Carl Zeiss Microscopy GmbH, Jena, Germany).

\section{Particle size distribution}

To determine the particle size distribution, the microspheres were dispersed in $1 \mathrm{~L}$ distilled water and measured with a Mastersizer 2000 from Malvern Instruments (Malvern, UK). The size and shape of the individual particles were determined by laser diffraction according to ISO 13320. The particle size distribution was determined by the intensity and deflection rate of a laser beam that passed through the sample. For evaluation, the Fraunhofer approximation was used. Next to particle size distribution, the parameters D10, D50, and D90 were obtained.

\section{Drug content and encapsulation efficiency of microspheres}

To determine the mass of totarol embedded into the microspheres as well as the entrapment efficiency, $5 \mathrm{mg}$ of microspheres were dissolved in a DMSO/water mixture. The sample was analyzed by UV/vis spectroscopy (BioTek EONTM; BioTek, Winooski, USA). For this purpose, a calibration curve was needed with concentrations ranging from $0.005 \mathrm{mg} / \mathrm{mL}$ to $0.075 \mathrm{mg} / \mathrm{mL}$ of totarol dissolved in a DMSO/ distilled water mixture. The measurement was carried out at $\lambda=285 \mathrm{~nm}$. All experiments were performed in triplicate.

$$
\text { Encapsulation }=\frac{\text { Actual drug loading }}{\text { Theoretical drug loading }} \times 100
$$

\section{In vitro drug release}

To determine the in vitro drug release, $10 \mathrm{mg}$ of totarolloaded microspheres were filled in $50 \mathrm{~mL}$ tubes containing $15 \mathrm{~mL}$ PBS and incubated for 24 hours at $37^{\circ} \mathrm{C}$. The microspheres were centrifuged, and the supernatant was used for the UV/vis spectroscopic analysis.

The measurements were carried out using a photometer (BioTek). Therefore, $400 \mu \mathrm{L}$ of the supernatant were transferred into UV-appropriate cuvettes and measured at $\lambda=285 \mathrm{~nm}$.

The centrifuged microspheres were resuspended in $15 \mathrm{~mL}$ PBS. After 24 hours, the released amount of totarol was analyzed. This step was repeated daily over the course of 90 days.

\section{In vitro antimicrobial activity}

\section{Minimal inhibitory concentration}

The minimal inhibitory concentration (MIC) was performed using the macrodilution method with different concentrations ranging from $4 \mu \mathrm{g}$ to $512 \mu \mathrm{g}$ totarol $/ \mathrm{mL}$ broth. The assay was carried out according to the standard M07-A9 recommended by the CLSI. An overnight culture of Staphylococcus aureus RN6390 was adjusted to 0.5 McFarland standard (BioMérieux, Marcy l'Etoile, France), which corresponds to $1.5 \times 10^{8}$ bacteria. The bacterial suspension was diluted 1:150 and divided in several tubes consisting of $1 \mathrm{~mL}$ each. Different concentrations of totarol dissolved in $20 \mu \mathrm{L}$ ethyl acetate were added into the different tubes, filled up to a volume of $2 \mathrm{~mL}$ and incubated at $37^{\circ} \mathrm{C}$. For the measurements after 24 hours, a photometer at $625 \mathrm{~nm}$ was used. As a control, $20 \mu \mathrm{L}$ ethyl acetate in a $2 \mathrm{~mL}$ bacteria suspension was applied. The final bacterial concentration in each tube was $5 \times 10^{5}$ bacteria. The MIC is defined as the lowest concentration of totarol that inhibited the initial inoculum.

\section{Agar diffusion test}

The totarol-loaded microspheres were tested for their characteristics on bacteria with the agar diffusion test (ADT). Therefore, $10 \mathrm{mg}$ totarol-loaded microspheres $(1.025 \mathrm{mg}$ 
pure totarol) were incubated for 7 days in $1 \mathrm{~mL}$ PBS. After this period, a filter paper disk with a diameter of $10 \mathrm{~mm}$ was soaked with $20 \mu \mathrm{L}$ of the supernatant and dried at air. An overnight culture of $S$. aureus RN6390 was adjusted to 0.5 McFarland standard. From this culture, $100 \mu \mathrm{L}$ were seeded on Mueller Hinton Blood agar plates. Subsequently, the disk was placed in the middle and incubated for 24 hours. The inhibition zone around the filter paper disk was investigated.

\section{Biofilm formation}

For investigation of biofilm formation, unloaded and totarolloaded microspheres were incubated in $1 \mathrm{~mL}$ of a $S$. aureus suspension, which was adjusted from an overnight culture to McFarland standard 0.5. The microspheres were incubated in this bacteria suspension for 24 hours and investigated by transmission electron microscope (Zeiss LIBRA 120 transmission electron microscope)

\section{Hemocompatibility}

Blood sampling procedure were approved by the ethic committee of the University of Tuebingen, Germany. The human whole blood was collected from three healthy blood donors who gave signed informed consent. Hemocompatibility testing was performed with fresh heparinized blood $(1.5 \mathrm{IU} / \mathrm{mL})$. Therefore, $0.1 \mathrm{mg} / \mathrm{mL}$ pure totarol, $1 \mathrm{mg} / \mathrm{mL}$ totarol-loaded microspheres, or $1 \mathrm{mg} / \mathrm{mL}$ unloaded microspheres were incubated with human blood for 60 minutes at $37^{\circ} \mathrm{C}$ in a dynamic rotation model. Afterward, various hemocompatibility markers were analyzed.

\section{Cytotoxicity of totarol-loaded and -unloaded microspheres}

The loaded and unloaded microspheres were also tested for their cytotoxic properties. Therefore, an MTT assay was carried out with the microspheres containing the highest amount of totarol $(92.36 \% \pm 0.99 \%)$.

BJ fibroblast and human embryonic kidney epithelial cells were seeded at a density of $1.5 \times 10^{5}$ cells/well in 12 -well culture plates with DMEM medium. After incubation for 24 hours at $37^{\circ} \mathrm{C}$ and $5 \% \mathrm{CO}_{2}$, the medium was removed, and the cells were washed once with PBS before adding fresh medium. Totarol-unloaded and -loaded microspheres with a weight of $0.5-2 \mathrm{mg} / \mathrm{mL}$ cell medium were added into the respective wells and incubated for 24 hours. Then, the medium was removed, and the cells were washed three times with PBS and RPMI medium without phenol red. In order to analyze the viability of the cells, an MTT assay was performed. Therefore, $30 \mu \mathrm{L}$ of a $0.5 \%$ MTT/PBS solution and $300 \mu \mathrm{L}$ of RPMI medium were added to the individual wells and incubated for 4 hours at $37^{\circ} \mathrm{C}$ and $5 \% \mathrm{CO}_{2}$. The medium was aspirated and replaced with $300 \mu \mathrm{L}$ DMSO followed by another incubation at $37^{\circ} \mathrm{C}$ and $5 \% \mathrm{CO}_{2}$ for 10 minutes. Afterward, $100 \mu \mathrm{L}$ were transferred to a 96-well plate and measured at $540 \mathrm{~nm}$ with the BioTek EONTM photometer. All experiments were carried out in triplicate.

\section{Statistics}

Data are depicted as mean with standard error of the mean (SEM). Data were analyzed using repeated measure one-way analysis of variance with Holm-Sidak's multiple comparison test to analyze differences between groups.

All analyses were performed using the statistical software package GraphPad Prism (Version 6; GraphPad Software, La Jolla, CA, USA). Statistical significance was defined as $P<0.05$.

\section{Results \\ Totarol purification}

Before purification by column chromatography, totarol had a purity of $\sim 68 \%$. The purification step significantly increased the purity. There was a clear difference in color between the dry purified and the original totarol, which is shown in Figure 1A and B. The TLC of purified totarol showed a clear spot in comparison with the non-purified totarol, which showed various bands (Figure 1C).

\section{Microsphere preparation}

The single emulsion evaporation method allows the reproducible production of loaded and unloaded microspheres.

In all sets of experiments, a constant amount of PLGA was used, whereas the totarol concentration varied between $1 \mathrm{~g}$ and $1.6 \mathrm{~g}$. Microspheres were examined under a light microscope and showed various sizes, but the same spherical shape (Figure 2A). Furthermore, no differences between totarol-loaded and -unloaded microspheres could be detected by scanning electron microscopy (Figure 2B).

\section{Particle size distribution}

The particle size distribution, which was determined at $\mathrm{D}(0.5)$, is shown in Figure 3. The D (0.5) for totarol-loaded PLGA microspheres was $147.21 \pm 3.45 \mu \mathrm{m}$, and $131.14 \pm 3.69 \mu \mathrm{m}$ for the unloaded PLGA microspheres (D (0.5) represents the average particle size distribution, which means that $50 \%$ of the particles are smaller and $50 \%$ are bigger than the specified value).

\section{Encapsulation efficiency}

The encapsulation efficiencies, as shown in Table 1, were largely independent of the amount of totarol used. The encapsulation efficiencies ranged from $84.72 \% \pm 6.68 \%$ to 

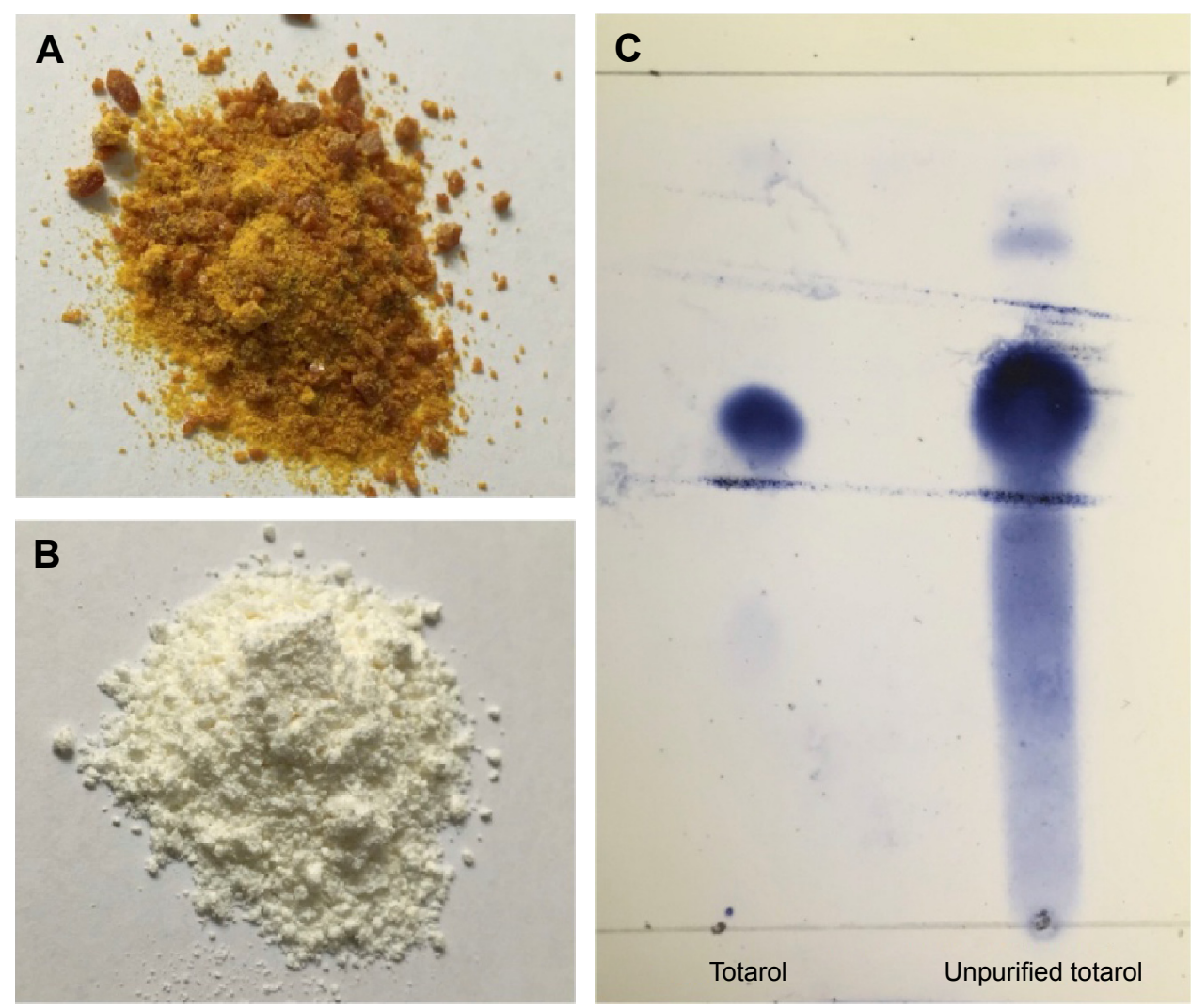

Figure I Purification of totarol.

Notes: Unpurified totarol (A) and purified totarol (B) via column chromatography followed by recrystallization. Analyses of unpurified and purified totarol using TLC (C). The purified totarol showed only one spot (left), whereas the unpurified totarol resulted in a large smear (right).

Abbreviation: TLC, thin layer chromatography.

$92.36 \% \pm 0.99 \%$. The drug content in $1 \mathrm{mg}$ microspheres ranged from $0.0830 \pm 0.0020 \mathrm{mg}$ to $0.1055 \pm 0.0025 \mathrm{mg}$.

\section{In vitro drug release}

Figure $4 \mathrm{~A}$ shows the kinetic drug release of $53.76 \%$ from totarol-loaded PLGA microspheres over a period of 90 days. The measured values of the unloaded PLGA microspheres were subtracted from the values of the totarol-loaded PLGA microspheres.

In order to detect the residual totarol content in the microspheres, they were dried, weighed, and analyzed. Figure 4B shows the amount of released totarol and the remaining totarol content of $45.07 \% \pm 2.72 \%$, which resulted in almost $100 \%$ recovery.
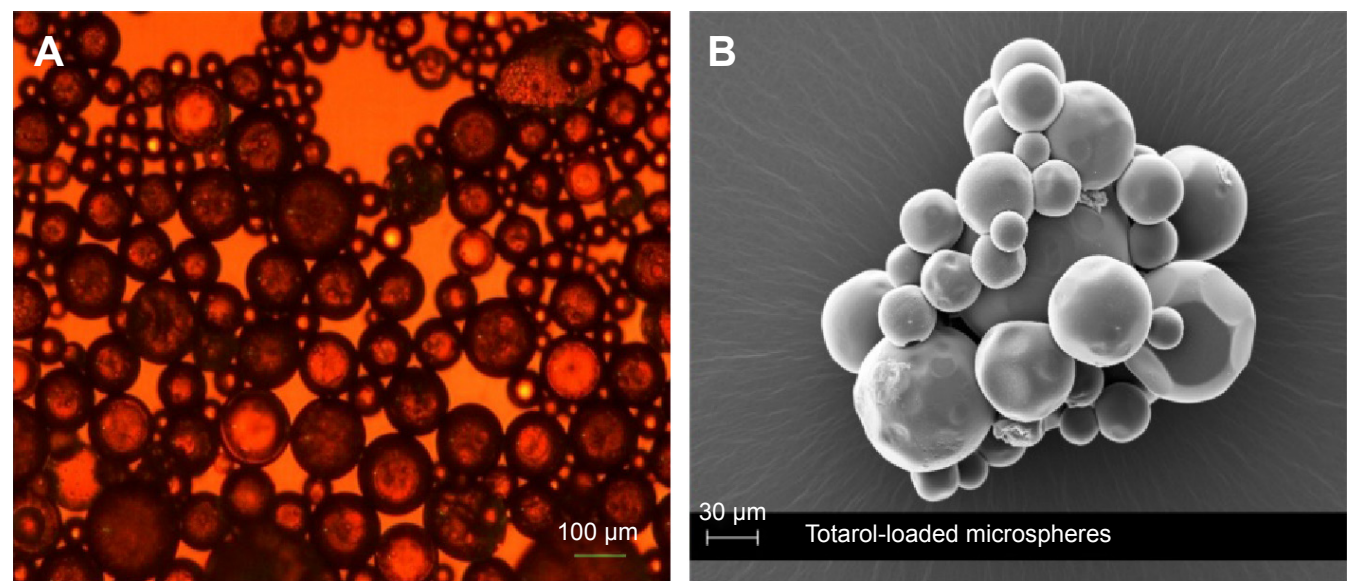

Figure 2 Microscopic analysis of totarol-loaded microspheres.

Notes: Light microscopic images of oil-in-water (o/w) emulsion-created totarol-loaded microspheres, which show spherical-shaped particles (A), 40× magnification. The scanning electron microscopic image confirms the round shape and shows also a very smooth surface (B), $600 \times$ magnification. 
A

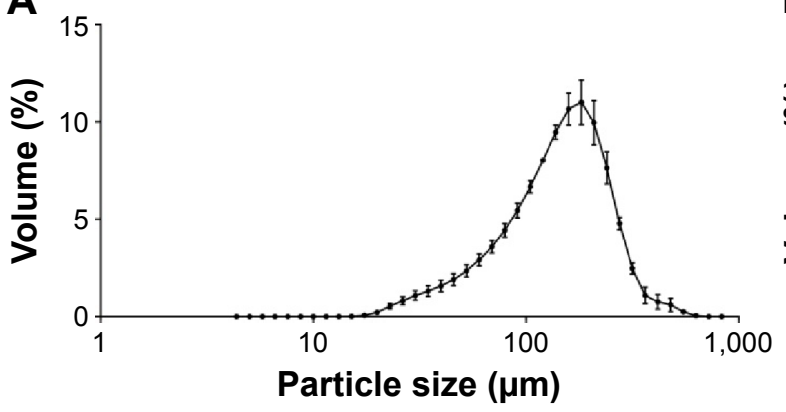

B

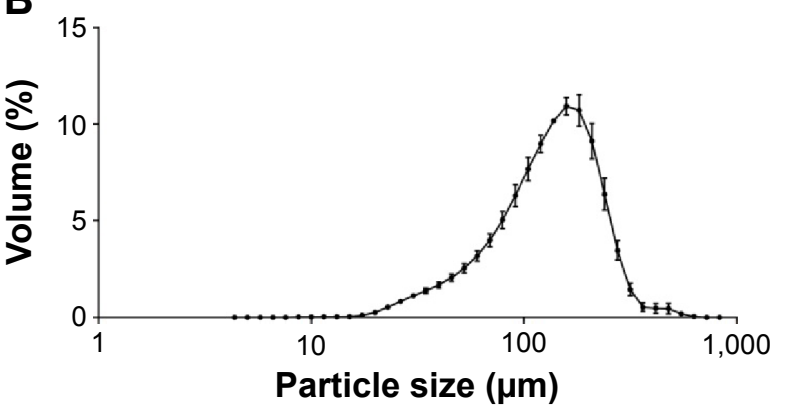

Figure 3 Measurement of particle size distribution of the generated microspheres.

Notes: Particle size distribution of totarol-loaded microspheres $(\mathbf{A})$ and unloaded microspheres (B). Both microspheres were created through the single emulsion evaporation method and measured via a Mastersizer 2000, which was carried out as triplets. The unloaded microspheres with a mean diameter of $131.140 \pm 3.69 \mu \mathrm{m}$ are smaller in comparison with totarol-loaded microspheres, which have a mean diameter of $147.205 \pm 3.45 \mu \mathrm{m}$.

\section{Antimicrobial activity of totarol}

The antimicrobial activity of totarol-loaded microspheres against $S$. aureus was evaluated by ADT and MIC.

The MIC measurement is shown in Figure 5A and B. The lowest concentration of totarol that completely inhibited bacterial growth after $24 \mathrm{~h}$ was $128 \mu \mathrm{g} / \mathrm{mL}$. The figure also shows that ethyl acetate had no significant influence on bacterial growth.

The ADT showed a small but clear inhibition zone around the filter paper disk (Figure 5C).

\section{Biofilm formation}

Unloaded and totarol-loaded microspheres were investigated on their properties to inhibit biofilm formation of $S$. aureus. After incubation in a bacteria suspension, biofilm formation could be detected at the surface of unloaded PLGA microspheres (Figure 6A) in comparison with totarol-loaded microspheres, where no biofilm formation could be detected (Figure 6B).

\section{Hemocompatibility}

The hemocompatibility of pure totarol, totarol-loaded microspheres and unloaded microspheres was investigated in a dynamic rotation model.

Our data show that totarol as well as the microspheres have no effect on the numbers of platelets (Figure 7A), leukocytes (Figure 7B) and erythrocytes (Figure 7C) when

Table I Amount of PLGA and totarol with the corresponding encapsulation efficiency and drug content

\begin{tabular}{llll}
\hline $\begin{array}{l}\text { PLGA } \\
(\mathbf{m g})\end{array}$ & $\begin{array}{l}\text { Totarol } \\
(\mathbf{m g})\end{array}$ & $\begin{array}{l}\text { Encapsulation } \\
\text { efficiency }(\%)\end{array}$ & $\begin{array}{l}\text { Content } \\
(\mathbf{m g} / \mathbf{m g})\end{array}$ \\
\hline $12,500.00$ & $\mathrm{I}, 000.00$ & $92.36 \pm 0.99$ & $0.083 \pm 0.002$ \\
$12,500.00$ & $\mathrm{I}, 200.00$ & $84.72 \pm 6.68$ & $0.084 \pm 0$ \\
$12,500.00$ & $\mathrm{I}, 600.00$ & $87.48 \pm 1.725$ & $0.106 \pm 0.003$ \\
\hline
\end{tabular}

Abbreviation: PLGA, poly(lactide-co-glycolide). compared to the control group. Furthermore, no effect was found investigating the hematocrit as well as hemoglobin levels (data not shown). With regard to activation of the coagulation and the complement cascades, the parameters thrombin-antithrombin III and SC5b-9 were measured. Thrombin-antithrombin III levels were not altered when compared to the control group indicating that totarol and the PLGA have no activating effect on the coagulation cascade (Figure 7D). Moreover, no statistical significance could be found analyzing the formation of the SC $5 b-9$ complex formation (Figure 7E). However, the data show that an increase of SC5b-9 concentration was induced by the unloaded and totarol-loaded microspheres, but not by pure totarol. This suggests that the PLGA itself might induce moderate complement activation.

\section{Cytotoxicity of totarol-loaded and -unloaded microspheres}

Figure $8 \mathrm{~A}$ and B shows the results of the MTT assay, which was carried out in order to detect cytotoxic effects of different amounts of loaded microspheres, unloaded microspheres, and totarol on BJ fibroblasts and human embryonic kidney cells. There was no significant difference between the microspheres, the totarol, and the control. All cells grew uniformly and did not display any uncharacteristic features; hence, cell viability was not altered by totarol.

\section{Discussion}

The aim of this study was to prepare totarol-loaded and -unloaded microspheres with the single emulsion evaporation method. These microspheres were compared to each other with respect to particle size distribution, cytotoxicity, and antimicrobial activity. Furthermore, the in vitro drug release and entrapment efficiency of totarol-loaded microspheres were investigated. 
A

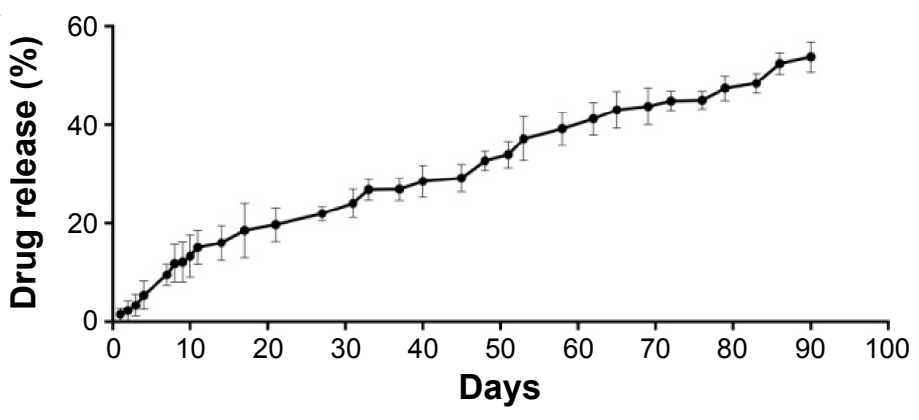

B

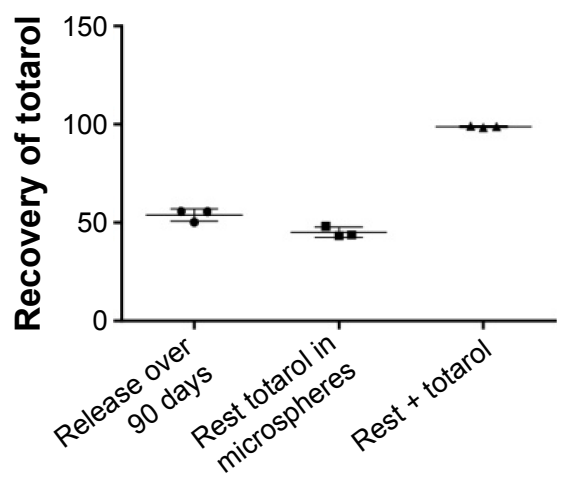

Figure 4 Investigation of the in vitro drug release from the manufactured microspheres.

Notes: (A) In vitro dissolution profile from totarol-loaded microspheres in PBS incubated at $37^{\circ} \mathrm{C}$ over 90 days. (B) Released and unreleased amount of totarol in PLGA microspheres after incubation in PBS for 90 days. In both diagrams, the data points represent the mean of three replicates.

Abbreviations: PBS, phosphate-buffered saline; PLGA, poly(lactide-co-glycolide).

First, it was important to check whether it was possible to purify the natural agent totarol, which was received in an impure state. A powerful tool to separate organic compounds is the combination of column chromatography and TLC. The implementation runs without major effort and is easier to use than high-performance liquid chromatography or gas chromatography.

The single emulsion evaporation method offers the possibility to produce microspheres loaded with active ingredients. This method is often used at a laboratory scale.
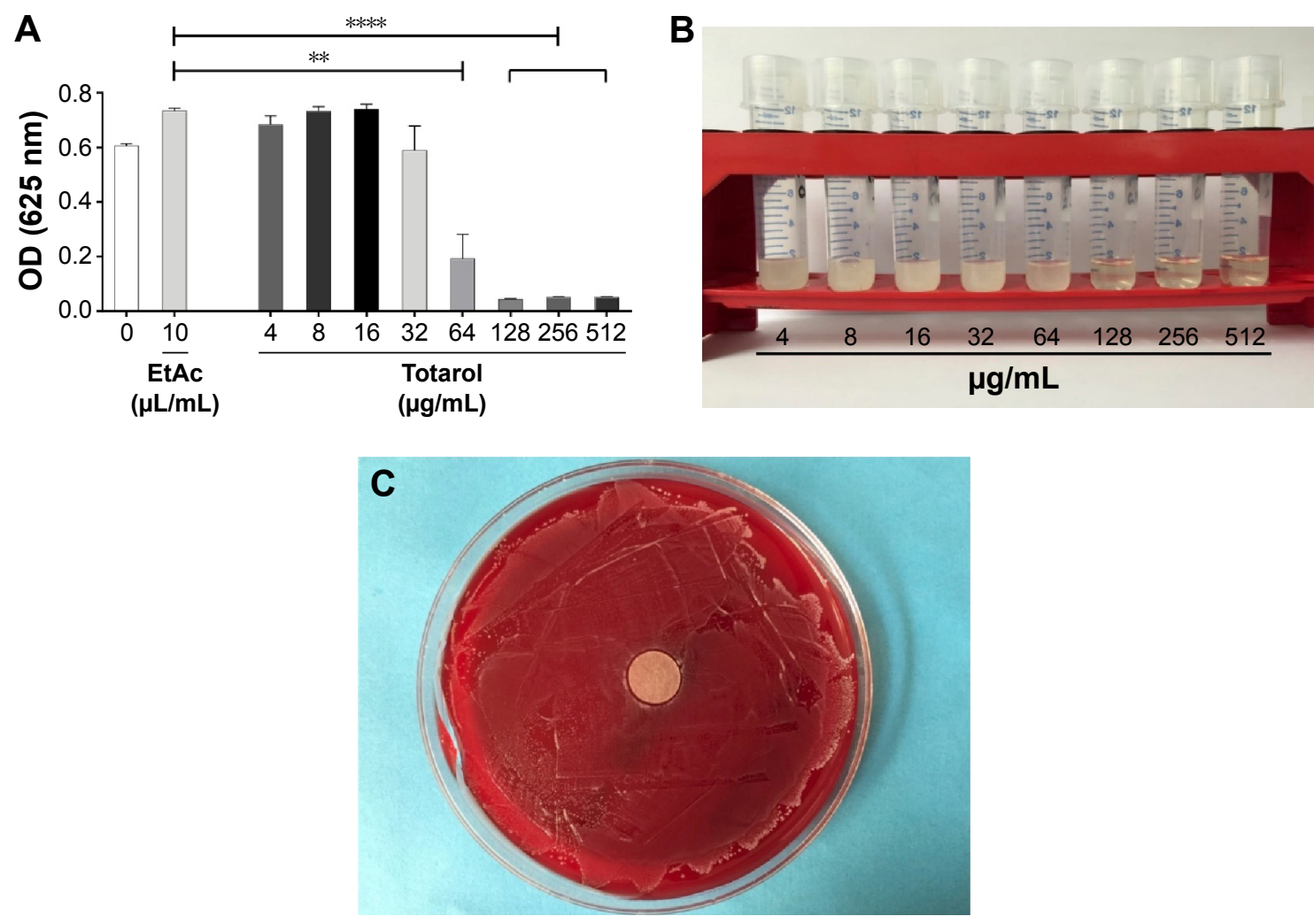

Figure 5 MIC of totarol against S. aureus RN6390.

Notes: The lowest totarol concentration against bacterial growth, after 24 hours incubation at $37^{\circ} \mathrm{C}$, was analyzed. The minimal inhibitory concentration was determined to be between $64 \mu \mathrm{g} / \mathrm{mL}$ and $128 \mu \mathrm{g} / \mathrm{mL}$ after 24 hours (A). MIC determination was realized using the marcodilution method with $5 \times 105$ bacteria in each tube (B); ** and ***** are significantly different from the negative control $(P<0.01$ and $P<0.000$ I). Results are presented as standard error of the mean ( $n=6)$. ADT test on Mueller Hinton blood agar (C). An amount of $10 \mathrm{mg}$ totarol-loaded microspheres were incubated in I mL PBS. A filter paper disk was soaked with $20 \mu \mathrm{L}$ of the supernatant after 7 days of incubation and placed on agar plate. The small but clear inhibition zone showed the antimicrobial activity.

Abbreviations: MIC, minimal inhibitory concentration; S. aureus, Staphylococcus aureus; PBS, phosphate-buffered saline; ADT, agar diffusion test; OD, optical density; EtAc, ethyl acetate. 

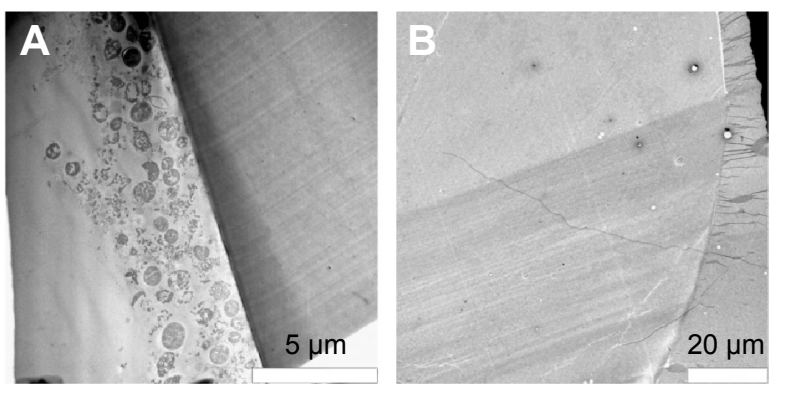

Figure 6 Transmission electron microscopic images of unloaded and totarol-loaded microspheres after incubation in bacteria suspension to investigate the biofilm formation.

Notes: (A) Unloaded microspheres, which show a great number of adhered bacteria on their surface (2,000 $\times$ magnification) and (B) totarol-loaded microspheres (3I5X magnification), where no bacteria were detectable on the surface.

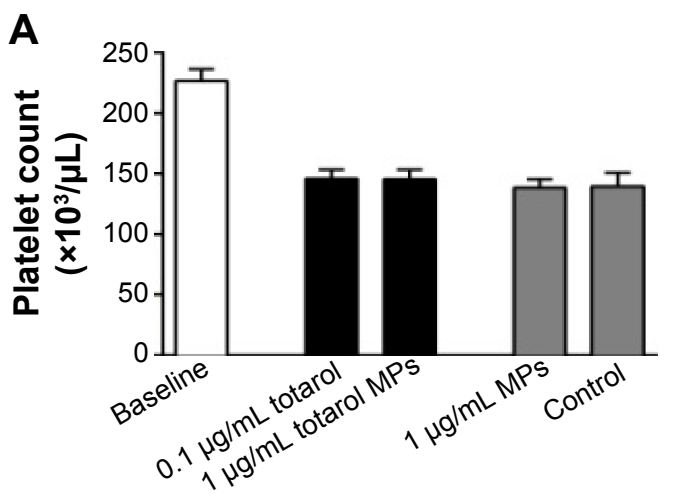

C

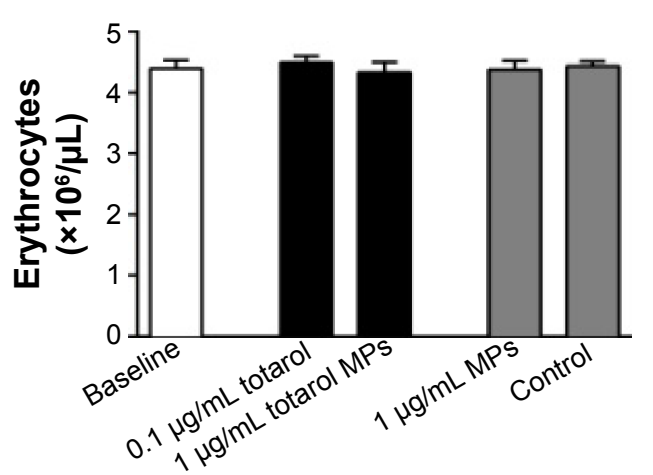

The loading of the active ingredient totarol, which takes place directly during the manufacturing process, can be achieved at a high efficiency. The microspheres were subsequently analyzed for their physical properties and tested in vitro for their cell toxicity and their antimicrobial effects against S. aureus. During the manufacturing, the toxic solvent chloroform was replaced by using the less toxic substance ethyl acetate. PLGA was chosen for its well-known and outstanding biocompatibility and its biological biodegradability. ${ }^{13}$ The low-molecular-weight polymer PLGA was used due to its slightly faster release kinetics, which were needed because totarol is not water-soluble.

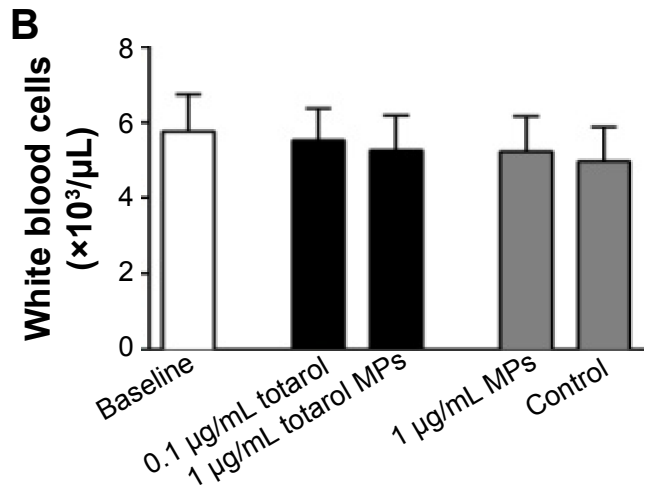

D

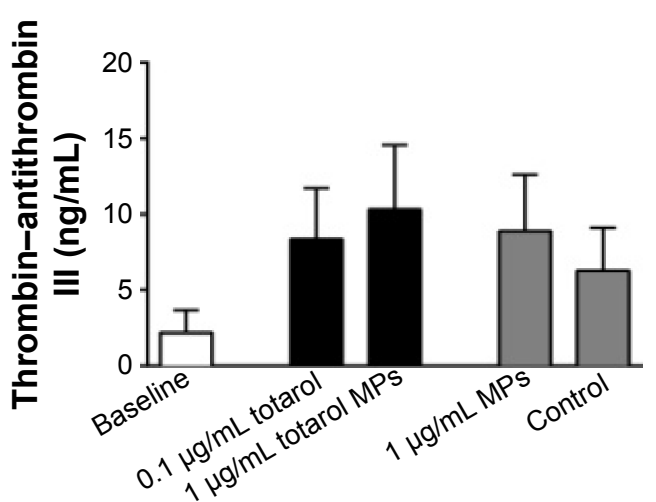

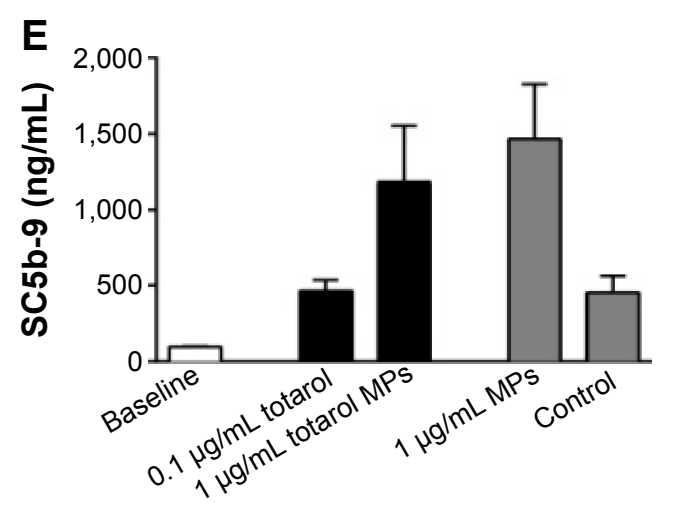

Figure 7 Hemocompatibility of totarol and totarol-loaded microspheres.

Notes: Hemocompatibility investigations exhibit no effect on (A) platelets, (B) leukocytes, and (C) erythrocytes in comparison to the control group. TAT III levels (D) were not altered, in comparison with the control. No significant difference was found by analyzing the SC5b-9 complex (E) concentration.

Abbreviations: TAT III, thrombin-antithrombin III; MPs, microspheres. 
A

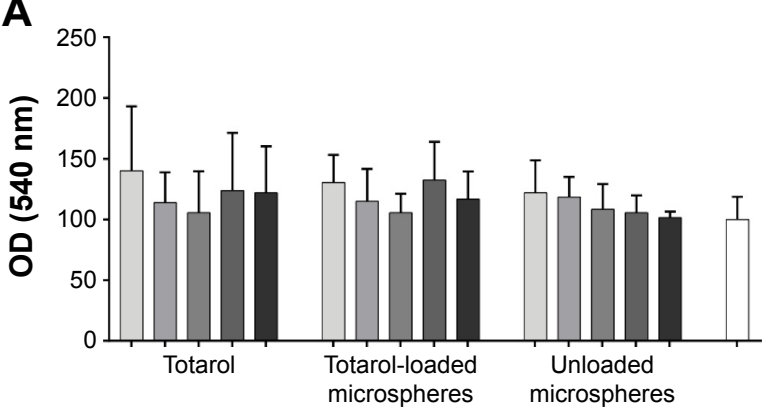

B

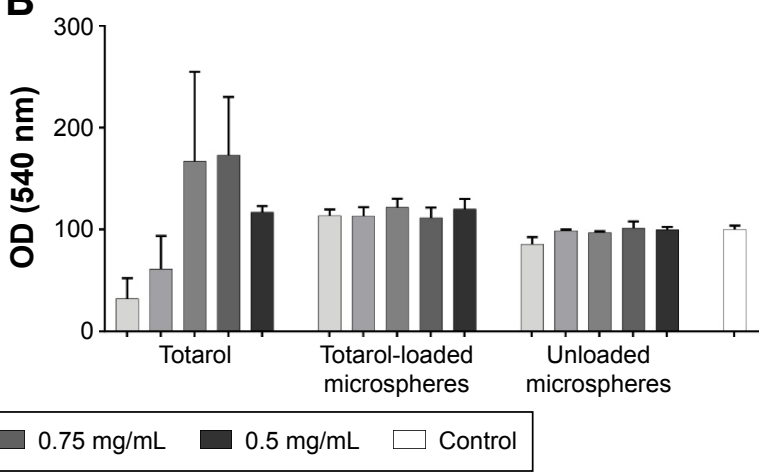

Figure 8 Different amounts of totarol, totarol-loaded microspheres, and unloaded microspheres have no influence on the viability of fibroblast (A) and epithelial (B) cells. Notes: MTT assay was performed after 24 hours of incubation at $37^{\circ} \mathrm{C}$ with $0.5 \mathrm{mg}, 0.75 \mathrm{mg}, \mathrm{I} \mathrm{mg}, 1.5 \mathrm{mg}$, or $2 \mathrm{mg}$ totarol, totarol-loaded microspheres, or unloaded microspheres per $\mathrm{mL}$ cell medium to evaluate the viability of the treated cells. Each bar represents the mean of at least three replicates.

Abbreviations: MTT, 3-(4,5-dimethylthiazol-2-yl)-2,5-diphenyltetrazolium bromide; OD, optical density.

Through this system, controlled drug release over 90 days could be realized. The release normally takes place via two different mechanisms: diffusion and erosion. Diffusion is controlled mainly by the initial release of the active ingredient, which is loosely bound to the surface of the microspheres. ${ }^{14}$ This effect, called burst release, could not be detected in this case, perhaps due to the solubility of totarol. The subsequent release is controlled mainly by the erosion of the microspheres. Here, cleavage of the ester bonds in the polymer backbone takes place. In contrast, individual fragments of the particles are cleaved out of the matrix due to erosion. The chemical degradation of PLGA is mediated by spontaneous hydrolysis of the ester bonds. ${ }^{13-15}$ After 90 days, a release of roughly $50 \%$ was detected. The crosscheck showed that the other $50 \%$ were still in the remaining fragments of the microspheres.

The produced microspheres were air-dried after being manufactured with the described emulsion process. Subsequently, the morphological study showed uniform spherical particles with smooth surfaces. The particles varied in size, which were determined accurately. The particle size distribution showed that the mean diameter was $147.21 \pm 3.45 \mu \mathrm{m}$ for the totarol-loaded microspheres and $131.14 \pm 3.69 \mu \mathrm{m}$ for the unloaded microspheres. The size of the microspheres had an influence on drug release and entrapment efficiency. By changing various parameters during the production process, the size could be influenced (data not shown). It was also observed that the entrapment efficiency decreased slightly but was still at a very high level with an average of $\sim 85 \%$.

To investigate the possible influence of the microspheres on cells, an MTT assay was performed. Ignatius and Claes $^{2}$ described that the $\mathrm{pH}$ value of the cell medium during incubation with PLGA does not change, which was not expected due to the PLGA degradation products.
Figure $8 \mathrm{~A}$ and $\mathrm{B}$ shows that there was no difference between the totarol-loaded microspheres and totarol. This suggests that even higher cache concentrations of totarol in microspheres did not have a statistically significant effect on fibroblasts and epithelial cells.

The antimicrobial effect of S. aureus was determined by ADT and MIC. Kubo et $\mathrm{al}^{9}$ described the antibacterial effects of different bacteria strains, which included S. aureus and methicillin-resistant S. aureus. Figure 5C shows a small but clear zone of inhibition around the totarol-soaked disk, indicating that totarol clearly inhibited bacterial growth, which was confirmed by the MIC test. The MIC (Figure 5A and B) determination for $S$. aureus was $64-128 \mu \mathrm{g} / \mathrm{mL}$ totarol and therefore higher than described. ${ }^{9}$

Further, the hemocompatibility investigations showed no influence of totarol on various blood parameters (Figure 7).

With regard to implant-related infections, which are a major cause of serious postoperative complications, coating of implants using, for example, the described totarol-loaded microspheres may be a promising strategy for prophylaxis. ${ }^{16}$ Perioperative coating of implants with antibacterial agents may decrease the incidence of implant-associated infections attributed mainly to the adherence of bacteria and consequent biofilm formation on the implant surface. Another important advantage of prophylactic prevention of biofilm formation on implants is the fact that already formed biofilms are extremely resistant against antimicrobial therapies. ${ }^{17-19}$

\section{Conclusion}

This study analyzed the feasibility of a drug delivery system consisting of totarol-loaded PLGA microspheres. No burst release was observed, and with only $53.76 \%$ totarol release within 90 days, we could clearly demonstrate its long-term 
drug release. In addition, the cytotoxicity tests confirmed that the microspheres had no influence on the viability of eukaryotic and epithelial cells. Additionally, bacterial tests confirmed a significant zone of inhibition around the totarolsoaked filter paper disk, reflecting the potential of preventing biofilm formation.

The combination of a PLGA drug delivery system with a natural antibacterial agent can be useful for local longterm treatment of bacterial infections without the use of antibiotics.

\section{Acknowledgments}

The bacterial strain (Staphylococcus aureus RN6390) was a kind gift from Professor Doctor Andreas Peschel (Interfaculty Institute for Microbiology and Infection Medicine, Tuebingen). We also thank Claudia Braun (Institute of Organic Chemistry) for help and advice with the chromatographic purification of totarol. The authors wish to thank Birgit Fehrenbacher (Department of Dermatology, University of Tuebingen, Germany) for her excellent technical assistance in imaging biofilm formation by transmission electron microscopy.

\section{Disclosure}

The authors report no conflicts of interest in this work.

\section{References}

1. Klose D, Siepmann F, Willart JF, Descamps M, Siepmann J. Drug release from PLGA-based microparticles: effects of the "microparticle:bulk fluid" ratio. Int J Pharm. 2010;383(1-2):123-131.

2. Ignatius AA, Claes LE. In vitro biocompatibility of bioresorbable polymers: poly(L, DL-lactide) and poly(L-lactide-co-glycolide). Biomaterials. 1996;17(8):831-839.

3. Shive MS, Anderson JM. Biodegradation and biocompatibility of PLA and PLGA microspheres. Adv Drug Deliv Rev. 1997;28(1):5-24.

4. Hu C, Feng H, Zhu C. Preparation and characterization of rifampicinPLGA microspheres/sodium alginate in situ gel combination delivery system. Colloids Surf B Biointerfaces. 2012;95:162-169.
5. Marquette S, Peerboom C, Yates A, et al. Stability study of full-length antibody (anti-TNF alpha) loaded PLGA microspheres. Int $J$ Pharm. 2014;470(1-2):41-50.

6. Jain RA. The manufacturing techniques of various drug loaded biodegradable poly(lactide-co-glycolide) (PLGA) devices. Biomaterials. 2000;21(23):2475-2490.

7. Lee SC, Oh JT, Jang MH, Chung SI. Quantitative analysis of polyvinyl alcohol on the surface of poly(D, L-lactide-co-glycolide) microparticles prepared by solvent evaporation method: effect of particle size and PVA concentration. J Control Release. 1999;59(2):123-132.

8. Vyslouzil J, Dolezel P, Kejdusova M, et al. Influence of different formulations and process parameters during the preparation of drugloaded PLGA microspheres evaluated by multivariate data analysis. Acta Pharm. 2014;64(4):403-417.

9. Kubo I, Muroi H, Himejima M. Antibacterial activity of totarol and its potentiation. J Nat Prod. 1992;55(10):1436-1440.

10. Muroi H, Kubo I. Antibacterial activity of anacardic acid and totarol, alone and in combination with methicillin, against methicillin-resistant Staphylococcus aureus. J Appl Bacteriol. 1996;80(4):387-394.

11. Haraguchi $\mathrm{H}$, Ishikawa $\mathrm{H}$, Kubo I. Antioxidative action of diterpenoids from Podocarpus nagi. Planta Med. 1997;63(3):213-215.

12. Haraguchi H, Oike S, Muroi H, Kubo I. Mode of antibacterial action of totarol, a diterpene from Podocarpus nagi. Planta Med. 1996;62(2): $122-125$.

13. Doan TV, Couet W, Olivier JC. Formulation and in vitro characterization of inhalable rifampicin-loaded PLGA microspheres for sustained lung delivery. Int J Pharm. 2011;414(1-2):112-117.

14. Raghuvanshi RS, Singh M, Talwar GP. Biodegradable delivery system for single step immunization with tetanus toxoid. Int J Pharm. 1993;93(1): R1-R5.

15. Berkland C, Kim K, Pack DW. PLG microsphere size controls drug release rate through several competing factors. Pharm Res. 2003;20(7): 1055-1062.

16. Schmidmaier G, Lucke M, Wildemann B, Haas NP, Raschke M. Prophylaxis and treatment of implant-related infections by antibiotic-coated implants: a review. Injury. 2006;37(suppl 2):S105-S112.

17. Xu Z, Lai Y, Wu D, et al. Antibacterial effects and biocompatibility of Titania nanotubes with octenidine dihydrochloride/poly(lactic-coglycolic acid). Biomed Res Int. 2015;2015:836939.

18. Mah TF, O'Toole GA. Mechanisms of biofilm resistance to antimicrobial agents. Trends Microbiol. 2001;9(1):34-39.

19. Gallo J, Holinka M, Moucha CS. Antibacterial surface treatment for orthopaedic implants. Int J Mol Sci. 2014;15(8):13849-13880.
Drug Design, Development and Therapy

\section{Publish your work in this journal}

Drug Design, Development and Therapy is an international, peerreviewed open-access journal that spans the spectrum of drug design and development through to clinical applications. Clinical outcomes, patient safety, and programs for the development and effective, safe, and sustained use of medicines are the features of the journal, which

\section{Dovepress}

has also been accepted for indexing on PubMed Central. The manuscript management system is completely online and includes a very quick and fair peer-review system, which is all easy to use. Visit http://www.dovepress.com/testimonials.php to read real quotes from published authors. 